\title{
Power Transformer Fault Diagnosis under Measurement Originated Uncertainties
}

\author{
Hui Ma, Chandima Ekanayake and Tapan K. Saha \\ The University of Queensland \\ Brisbane, Australia
}

\begin{abstract}
This paper addresses the problem of diagnosing the fault symptoms of power transformers with measurement originated uncertainties, which arise from the imprecision of samples (i.e. due to noises and outliers) and the effect of class imbalance (i.e. samples are unequally distributed between different fault types) in a training dataset used to identify different fault types. Two fuzzy support vector machine (FSVM) algorithms namely fuzzy $c$-means clustering-based FSVM (FCM-FSVM) and kernel fuzzy $c$-means clustering-based FSVM (KFCM-FSVM) have been applied in this paper to deal with any noises and outliers in training dataset. In order to reduce the effect of class imbalance in training dataset, two approaches including betweenclass weighting and random oversampling have been adopted and integrated with FCM-FSVM and KFCM-FSVM. The case studies show that KFCM-FSVM algorithm and its variants have consistent tendency to attain satisfied classification accuracy in transformer fault diagnosis using dissolved gas analysis (DGA) measurements.
\end{abstract}

Index Terms - Condition monitoring, dissolved gas analysis, measurement originated uncertainties, and power transformer.

\section{INTRODUCTION}

DISSOVLED gas analysis (DGA) has been widely adopted by the electricity utilities for power transformer fault diagnosis [1-3]. The conventional DGA interpretation methods make use of concentrations or relative proportions of byproduct gases such as hydrogen, methane, acetylene, ethylene, ethane, carbon monoxide and carbon dioxide to detect thermal and discharge faults occurring in transformers. In the key gas method, several gas contents are evaluated to detect four types of faults including oil overheating, paper overheating, partial discharge, and arcing. In the ratio based methods such as Dörnenburg Ratios, Rogers Ratios, Duval Triangle and IEC/IEEE standards, the diagnosis is made by computing several key gas ratios and mapping these ratios to the predefined fault patterns.

Although widely adopted in utilities, the above conventional DGA interpretation methods still suffer some limitations. The diagnosis criteria may vary amongst utilities and there might be discrepancies among diagnoses by using different ratios. Sometimes the conventional methods may not be able to provide an interpretation for every possible combination of ratio values and give an explicit diagnosis. To address these limitations artificial intelligence (AI) techniques have been developed to identify different fault conditions of transformers [4-14]. One of the key advantages of AI

Manuscript received on 12 May 2012, in final form 14 August 2012. techniques is the utilization of historic DGA dataset, i.e. the DGA measurement records of which the transformers fault conditions are already known. By exploring the underlying mathematical relationship between these DGA records and the corresponding fault types, the AI algorithms can predict the fault type of any new DGA measurement for a transformer of interest. In AI algorithms the process of exploiting the mathematical relationship is called the training process and the historic DGA dataset is referred to as the training dataset.

In the training process, it is normally assumed that the fault type is known with certainty for each DGA record. However, due to the complexity of the transformer insulation system, imprecision of the measurement system, influence from any environmental noise and interference, limitation of human interpretation on the measurement data, and possible presence of multiple faults, there is a certain degree of inaccuracy and uncertainty in determining the fault type for each DGA record of training dataset. As a result, some DGA records can be fully assigned to one of the fault types while others may not be assigned exactly to one of the fault types. Moreover, there exist some data points which are distant from the rest of the data points. Although these data points (outliers) are not error data, they may also cause difficulties in the training process. On the other hand, the occurrence rate of some types of faults in power transformer is rather low, which implies that the DGA records of these type of faults are very limited and the training dataset may exhibit an unequal distribution between 
different fault types (i.e. class imbalance problem [15]). Figure 1 illustrates the concept of noise/outliers and class imbalance in the training dataset.

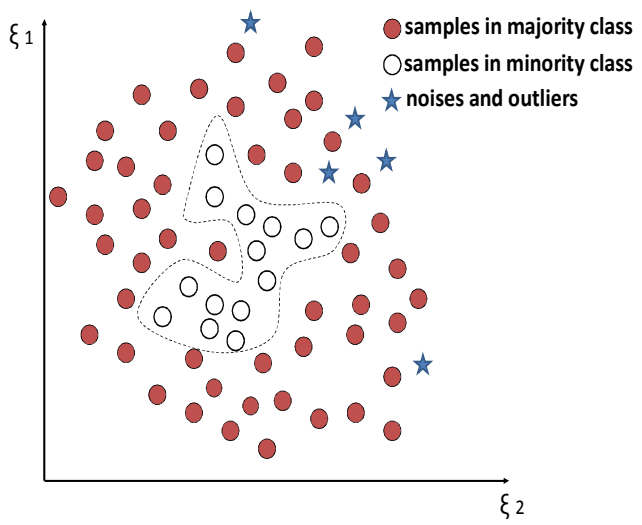

Figure 1. Illustration of measurement originated uncertainties.

(This figure shows a two dimensional dataset with two classes of samples. $\zeta_{1}$ and $\zeta_{2}$ represent the two axes. The majority class has a large number of samples and the minority class has a relatively small number of samples.)

In this paper the above phenomena of noises and outliers and the class imbalance in training dataset are referred to as measurement originated uncertainties. Obviously these uncertainties will introduce considerable challenges in reaching a correct decision of fault type for any new DGA measurement of the transformer under investigation. Therefore, appropriate techniques have to be employed to carry out the necessary processing of training dataset with measurement originated uncertainties.

This paper applies clustering based fuzzy support vector machine (FSVM) algorithms to deal with the noises and outliers of the training dataset in recognizing different fault types in transformers. These are: (a) Fuzzy c-means clustering-FSVM (FCM-FSVM); (b) Kernel fuzzy $c$-means clustering-FSVM (KFCM-FSVM). FSVM is a variant of support vector machine (SVM) and it is effective in dealing with noises and outliers present in the training dataset [16-19]. In FCM-FSVM and KFCM-FSVM, the samples (e.g. DGA records) in training dataset are assigned with different weights. The samples that are outliers or noises corrupted will be assigned a smaller weight by the algorithms and consequently treated less importantly in the training process. In order to reduce the effect of class imbalance of training dataset, two approaches namely between-class weighting and random oversampling are adopted and integrated with FCM-FSVM and KFCM-FSVM.

The paper is organized as follows. Section 2 provides a brief literature review on AI based DGA interpretation methods. Section 3 presents the mathematic formulation of FSVM. Section 4 details the FCM-FSVM and KFCM-FSVM, including data clustering, weights calculation and implementation. Section 5 presents the approaches for dealing with the class imbalance problem in training dataset. Section 6 presents the case studies and Section 7 concludes the paper.

\section{AI TECHNIQUES FOR TRANSFORMER FAULT DIAGNOSIS BASED ON DGA}

Over the past ten years AI based techniques have been extensively explored for transformer fault diagnosis, including expert system, fuzzy inference system, artificial neural networks, wavelet transformation and hybrid system ...etc [4 -14]. This section provides a brief review on AI techniques for transformer fault diagnosis based on DGA measurements.

The expert system constructs diagnosis rules upon a knowledge base, which may include transformers' information and experts' experience regarding fault symptoms [4]. Fuzzy inference system makes use of fuzzy if-then rules to incorporate the experts' knowledge $[5,6]$. The major drawbacks of the expert and fuzzy inference system for DGA interpretation are that their performances depend on the completeness of the knowledge base and they cannot make automatic adjustments (i.e. re-computing the weights) when new knowledge becomes available.

Artificial neural network (ANN) has also been applied to transformer diagnosis [7-9]. ANN can learn hidden relations between DGA data and the faults occurring in transformers. The major advantage of ANN over the expert and fuzzy inference system is that ANN can incrementally extend its knowledge base by incorporating newly available data. However, ANN still suffers some drawbacks such as having no explanation ability, slow convergence, and requiring a relatively large number of training data.

Attempts have been made to integrate ANN with expert or fuzzy inference system to improve overall diagnosis accuracy. Wang et al combined ANN and expert system to take advantages of the self-learning and non-linear mapping capability of ANN and the explicit knowledge representation capability of expert system [10]. Naresh et al proposed a neural fuzzy approach which makes use of fuzzy logic's capability in uncertainty representation and ANN's capability in learning [11].

Huang et al integrated fuzzy logic and neural net with an evolutionary algorithm because of its capability in solving optimization problems [12, 13]. In [12], the hybrid evolutionary-fuzzy logic algorithm was developed to solve a mixed-integer combinatorial optimization problem. This hybrid algorithm automatically modified 24 if-then rules and adjusted the weights of the fuzzy logic system. In [13], a hybrid neural net and evolutionary algorithm was developed. This hybrid algorithm makes use of the nonlinear mapping capability of neural net and the global search capability of evolutionary algorithm. As a result, it is able to overcome some problems of conventional neural network, especially the slow convergence and the needs of manually determining the network structure and parameters.

Different AI techniques have their own merits and drawbacks for transformer fault diagnosis. There are still avenues for further research, especially the applicability and adaptability of AI techniques when they are applied at different utilities. This paper adopts support vector machine (SVM) technique for transformer fault diagnosis. SVM technique has been proved as a powerful tool for solving classification problems [16-19]. Especially, this paper investigates clustering based fuzzy support vector machine to 
deal with the noises and outliers as well as the class imbalance problem in training dataset for transformer fault diagnosis using DGA measurements.

\section{FUZZY SUPPORT VECTOR MACHINE}

The SVM algorithm adopts kernel-based technique to transform the training samples from the original input space into a higher dimensional feature space, in which a hyperplane that maximizes the margin between different groups of samples is sought (Figure 2). This margin maximization is formulated as a quadratic programming (QP) problem and solved by introducing the Lagrange multipliers [16, 17].

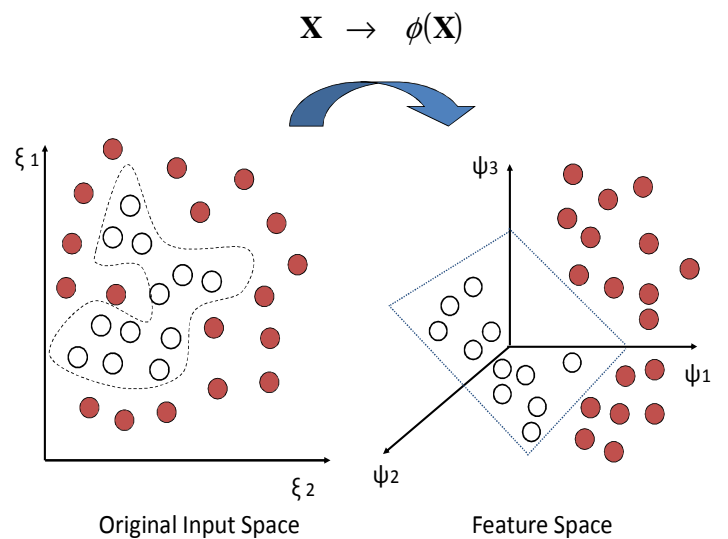

Figure 2. Illustration of data transformation in SVM.

However one of the main drawbacks of the normal SVM is that all samples in the training dataset are treated uniformly. Thus, the normal SVM lacks the ability to deal with the training dataset with noises and outliers $[18,19]$. To solve this problem this paper resorts to fuzzy support vector machine (FSVM). Differing from general fuzzy classification which involves fuzzification, defuzzification and fuzzy reasoning, FSVM assigns different weights for different samples in the training dataset by taking into account their relevance and then incorporates the weights into the normal SVM training process to reduce the effect of noise and outliers. The mathematic formulation of FSVM is briefly reviewed as follows.

It is assumed that there are $N$ samples $\mathbf{X}=\left[\mathrm{x}_{1}, \ldots, \mathrm{x}_{N}\right]$ in the training dataset and each sample belongs to one of the $T$ independent classes $\left\{y_{1}, \ldots, y_{T}\right\}$. In FSVM each sample within the training dataset is assigned a weight and these weights are added to the original training dataset, which then becomes $\left\{\mathrm{x}_{k}, y_{k}, \rho_{k}\right\}_{k=1}^{N}$, where $0<\rho_{k}<1$ are the weights describing the degree of $\mathrm{x}_{k}$ belonging to $y_{k}$. This paper adopts fuzzy $c$-means clustering (FCM) and kernel fuzzy $c$ means clustering (KFCM) algorithm to facilitate the computation of samples weights. The details of weights computing will be discussed in the next section.

FSVM algorithm starts with transforming the samples $\mathbf{x}$ from the original input space $\Re^{m}$ to a higher dimensional feature space $\Omega$ through a nonlinear function $\phi(\mathbf{X})$. Then it searches for an optimal separation hyperplane in that feature space by solving the following quadratic programming (QP) problem [18]:

$$
\begin{aligned}
\text { Minimize } & \frac{1}{2} \mathbf{w}^{T} \mathbf{w}+C \sum_{k=1}^{N} \rho_{k} \xi_{k} \\
\text { Subject to } & y_{k}\left(\mathbf{w}^{T} \cdot \phi\left(\mathrm{x}_{k}\right)+b\right) \geq 1-\xi_{k} \quad k=1, \ldots, N \\
\text { and } & \xi_{k} \geq 0 \quad k=1, \ldots, N
\end{aligned}
$$

In the above equations, the pair $(\mathrm{w}, b)$ defines the separation hyperplane, in which $\mathrm{w}$ is a normal vector of the hyperplane and $b$ is a bias. $C$ is the regularization parameter to balance the margin maximization and misclassification, and $\xi_{k} \geq 0$ are the error terms due to the misclassifications. The above QP problem can be transformed into its dual form [16], [18]:

$$
\text { Maximize } \sum_{k=1}^{N} \alpha_{k}-\frac{1}{2} \sum_{k=1}^{N} \sum_{j=1}^{N} \alpha_{k} \alpha_{j} y_{k} y_{j} K\left(\mathrm{x}_{k}, \mathrm{x}_{j}\right)
$$

$$
\begin{aligned}
& \text { Subject to } \quad \sum_{k=1}^{N} \alpha_{k} y_{k}=0 \\
& \text { and } 0 \leq \alpha_{k} \leq \rho_{k} C, k=1, \ldots, N
\end{aligned}
$$

where $\alpha_{k}$ is the Lagrange multiplier, and $K\left(\mathrm{x}_{k}, \mathrm{x}_{j}\right)$ is the kernel function in the form of $K\left(\mathrm{x}_{k}, \mathrm{x}_{j}\right)=\phi\left(\mathrm{x}_{k}\right)^{T} \phi\left(\mathrm{x}_{j}\right)$.

After obtaining the maximized hyperplane, FSVM can predict the class label for a new sample $\mathrm{X}$ as

$$
y=\operatorname{sgn}\left[\sum_{k=1}^{N} \alpha_{k} y_{k} K\left(\mathrm{x}_{k}, \mathrm{x}\right)+b\right]
$$

\section{FUZZY CLUSTERING BASED FUZZY SUPPORT VECTOR MACHINE}

To decide the weights for samples in the training dataset for the above FSVM, two algorithms namely fuzzy $c$-means clustering (FCM) and kernel fuzzy $c$-means clustering (KFCM) are adopted. Both algorithms group samples based on their similarity, in which similar samples (i.e. having the same type of fault condition in transformer) form a cluster (group). The samples weights calculation is performed in two steps: (1) Clusters formation. In this step, the centers of each cluster and samples within each cluster are determined. Firstly, the FCM (KFCM) is performed to decide a number of cluster centers as well as the samples membership values to each center. Then, each sample is assigned to one cluster, to which the sample has the largest membership value. (2) Samples weights computation. This step computes a sample's weight based on its Euclidean distance to the center of its belonged cluster. An exponentially decaying function (an inverse of Euclidean 
distance between a sample and its cluster center, refer to Equation 18) is used to compute the final weights for samples in each cluster.

This section provides a brief review of FCM and KFCM and the implementation of the resultant FCM-FSVM and KFCM-FSVM algorithm.

\subsection{FCM ALGORITHM}

In FCM algorithm, the forming of clusters is achieved by minimizing a quadratic objective function. The formed clusters are represented in the format of a fuzzy partition matrix and a collection of several cluster centers.

It is assumed that there are $N$ samples $\mathbf{X}=\left[\mathrm{x}_{1}, \ldots, \mathrm{x}_{N}\right]$, which belong to $c(2 \leq c<N)$ clusters. Now the task for FCM is to decide the cluster centers, which is accomplished through the minimization of a quadratic objective function as follows [20]:

$$
\begin{array}{ll}
\text { Minimize } & J(\mathrm{X} ; \mathrm{U}, \mathrm{V})=\sum_{i=1}^{c} \sum_{j=1}^{N}\left(u_{i j}\right)^{m}\left\|\mathrm{x}_{j}-\mathrm{v}_{i}\right\|^{2} \\
\text { Subject to } & \sum_{i=1}^{c} u_{i j}=1, \forall j=1,2, \ldots N
\end{array}
$$

where $\mathrm{V}=\left[\mathrm{v}_{1}, \mathrm{v}_{2}, \ldots, \mathrm{v}_{c}\right], \mathrm{v}_{i} \in \mathrm{R}^{n}$ is the cluster centers to be decided, $U=\left[u_{i j}\right]$ is the partition matrix, in which $u_{i j}$ is the membership of the $j$-th sample with the $i$-th group, $\left\|\mathrm{x}_{j}-\mathrm{v}_{i}\right\|^{2}>0, \forall i, j$ is a squared distance norm, and $m$ is the fuzziness coefficient.

The pair of $(\mathrm{U}, \mathrm{V})$ that minimizes the above objective function can be obtained through the following iterations [20]:

$$
\begin{gathered}
\mu_{i j}=\frac{1}{\sum_{k=1}^{c}\left(\frac{\left\|\mathrm{x}_{j}-\mathrm{v}_{i}\right\|}{\left\|\mathrm{x}_{j}-\mathrm{v}_{k}\right\|}\right)^{2 /(m-1)}}, 1 \leq i \leq c, 1 \leq j \leq N \\
\mathrm{v}_{i}=\frac{\sum_{j=1}^{N} \mu_{i j}^{m} \mathrm{x}_{j}}{\sum_{j=1}^{N} \mu_{i j}^{m}}, 1 \leq i \leq c
\end{gathered}
$$

In the implementation, FCM algorithm starts with a random partition matrix. The membership value $u_{i j}$ is updated to $u_{i j}^{\text {new }}$ after each iteration and the iterations will be terminated if $\max _{i, j}\left|u_{i j}-u_{i j}^{\text {new }}\right|<\mathcal{E}$, where $\mathcal{E}$ is a small positive value.

\subsection{KFCM ALGORITHM}

In the above FCM algorithm, the squared-norm is adopted as the similarity measure for samples clustering. However this may only be effective in clustering "spherical" datasets. To cater for clustering more general datasets, a number of variants of FCM have been proposed [21-23]. Amongst these variants, the kernelized version of FCM, named as kernel
FCM (KFCM) performs better than the "standard" FCM [22, 23].

Instead of using Euclidean norm as the similarity measure, KFCM adopts a more robust kernel induced similarity measure. This will help the resultant KFCM-FSVM algorithm effectively deal with noises and outliers and achieve better classification accuracy than FCM-FSVM algorithm does. The numeric experiments results of cases studies (Section 6) will show that the KFCM-FSVM is more robust than FCM-FSVM in transformer fault diagnosis based on DGA measurements. KFCM minimizes the following objective function [22]:

$$
\begin{gathered}
\operatorname{Minimize} J(\xi(\mathrm{x}) ; \mathrm{U}, \mathrm{V})=\sum_{i=1}^{c} \sum_{j=1}^{N}\left(u_{i j}\right)^{m}\left\|\xi\left(\mathrm{x}_{j}\right)-\xi\left(\mathrm{v}_{i}\right)\right\|^{2} \\
\text { Subject to } \quad \sum_{i=1}^{c} u_{i j}=1, \forall j=1,2, \ldots N
\end{gathered}
$$

where $\xi: \mathrm{x} \rightarrow \xi(\mathrm{x})$ defines a nonlinear transformation from original input space to a high dimensional feature space. In Equation (12), the norm is in the form of [22, 23]:

$$
\left\|\xi\left(\mathrm{x}_{j}\right)-\xi\left(\mathrm{v}_{i}\right)\right\|^{2}=K\left(\mathrm{x}_{j}, \mathrm{x}_{j}\right)+K\left(\mathrm{v}_{i}, \mathrm{v}_{i}\right)-2 K\left(\mathrm{x}_{j}, \mathrm{v}_{i}\right)
$$

where $K(\mathrm{x}, \mathrm{y})=\xi(\mathrm{x})^{T} \xi(\mathrm{y})$ is a kernel function. In this paper the Gaussian kernel $K(\mathrm{x}, \mathrm{y})=\exp \left(-\|\mathrm{x}-\mathrm{y}\|^{2} / \sigma^{2}\right)$ is adopted, which has the property of $K(\mathrm{x}, \mathrm{x})=1$. By adopting the Gaussian kernel, the above objective function, i.e. Equation (12) can be rewritten as:

$$
\hat{J}(\xi(\mathrm{x}) ; \mathrm{U}, \mathrm{V})=2 \sum_{i=1}^{c} \sum_{j=1}^{N}\left(u_{i j}\right)^{m}\left(1-K\left(\mathrm{x}_{j}, \mathrm{v}_{i}\right)\right)
$$

By adopting Lagrange multipliers, the iterations for calculating the partition matrix $\mathrm{U}$ and group centers $\mathrm{V}$ are:

$$
\begin{gathered}
\mu_{i j}=\frac{\left(1 /\left(1-K\left(\mathrm{x}_{j}, \mathrm{v}_{i}\right)\right)\right)^{1 /(m-1)}}{\sum_{k=1}^{c}\left(1 /\left(1-K\left(\mathrm{x}_{j}, \mathrm{v}_{k}\right)\right)\right)^{1 /(m-1)}}, 1 \leq i \leq c, 1 \leq j \leq N \\
\mathrm{v}_{i}=\frac{\sum_{j=1}^{N} \mu_{i j}^{m} K\left(\mathrm{x}_{j}, \mathrm{v}_{i}\right) \mathrm{x}_{j}}{\sum_{j=1}^{N} \mu_{i j}^{m} K\left(\mathrm{x}_{j}, \mathrm{v}_{i}\right)}, 1 \leq i \leq c
\end{gathered}
$$

The KFCM algorithm starts with a random partition matrix. The partition matrix and group centers are then updated iteratively until the termination value $\mathcal{E}$ is reached.

\subsection{FCM-FSVM AND KFCM-FSVM ALGORITHM}

After forming clusters (i.e. determining the cluster centers and assigning samples to the clusters based on their membership values), the sample weights $\rho\left(\mathrm{x}_{k}\right)$ of FSVM is computed as an exponentially decaying function [24]:

$$
\rho\left(\mathrm{x}_{k}\right)=\frac{2}{1+\exp \left(\beta d_{k}^{\text {cen }}\right)} \quad \beta \in[0,1]
$$


where $d_{k}^{c e n}=\left\|\mathrm{x}_{k}-\mathrm{v}_{i}\right\|^{1 / 2}$ is the Euclidean distance between the sample $\mathrm{X}_{k}$ and its cluster center $\mathrm{v}_{i}$, and $\beta$ determines the steepness of the decay. According to equation (18), the samples closer to the cluster center are treated as more credible and assigned higher weights while the samples away from the center are treated as less credible and assigned lower weights. Once the weight is computed for each sample in the training dataset, the FSVM is invoked to obtain a nonlinear classifier and finally can make fault classification on the transformer of interest (e.g. testing dataset). The overall procedure of FCM-FSVM and KFCM-FSVM is depicted in Figure 3 .

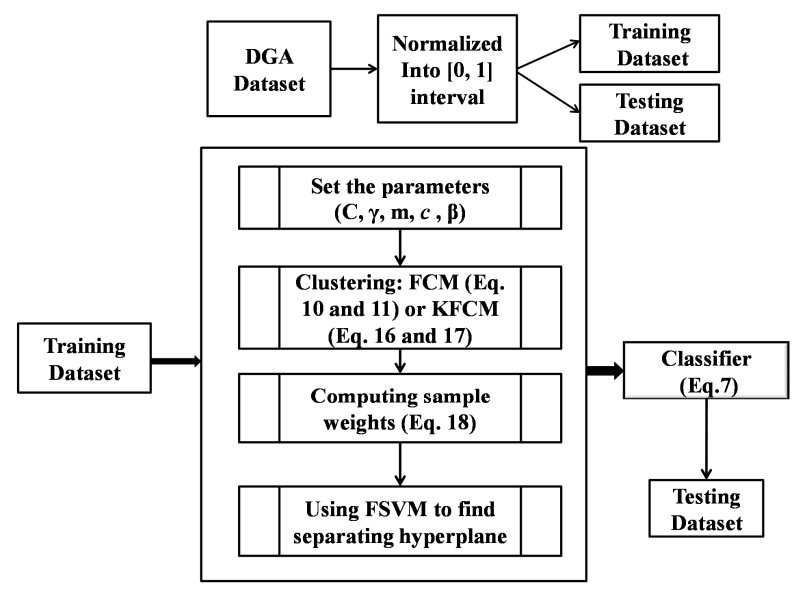

Figure 3. Flowchart of FCM-FSVM and KFCM-FSVM algorithm.

\section{DEALING WITH CLASS IMBALANCE}

Both FCM-FSVM and KFCM-FSVM can deal with the problems of noises and outliers in training dataset. However, the performance of both algorithms could still be jeopardized by the effect of class imbalance in training dataset. FCMFSCM and KFCM-FSVM may generate a biased model, which is in favor of the majority classes (i.e. the fault types having large number of samples) and provides lower classification accuracy on the minority classes (i.e. the fault types having small number of samples).

The problem of training algorithms using the class imbalanced dataset has attracted more attentions and many approaches have been proposed [15, 24]. This paper adopts between-class weighting and random oversampling approach for handling the class imbalance problem. These two approaches will be integrated with FCM-FSVM and KFCMFSVM to provide these two algorithms with the capability to reduce the effect of class imbalance without significantly affecting their capabilities in dealing with noises and outliers.

\subsection{BETWEEN-CALSS WEIGHTING}

In this approach, a between-class weight is introduced to combat the class imbalance problem: a lower weight is assigned to all samples in the majority class and a higher weight is assigned to all samples in the minority class. These weights should not be confused with the weights discussed in the previous section, which are assigned to samples based on their trustworthiness with the class to which they are belonged.

The assignment of between-class weights can be exemplified with a following two classes classification. Let $\eta^{+}$represents the between-class weight for all samples in the minority class and $\eta^{-}$represents the between-class weight for all samples in the majority class. The between-class weights can be assigned as $\eta^{+}=1$ and $\eta^{-}=1 / \eta$, where $\eta=\frac{\text { number of samples in majority calss }}{\text { number of samples in minority class }}$.

Combing the above between-class weights with the weights computed using FCM or KFCM algorithm (refer to Section 4), the overall weights for each sample in the training dataset becomes:

$$
\begin{aligned}
& \omega_{k}^{+}=\rho\left(\mathrm{x}_{k}\right) \eta^{+} \\
& \omega_{k}^{-}=\rho\left(\mathrm{x}_{k}\right) \eta^{-}
\end{aligned}
$$

where $\rho\left(\mathrm{x}_{k}\right)$ is the weights computed with either FCM or KFCM. $\omega_{k}^{+}$and $\omega_{k}^{-}$are the overall sample weights for the samples in the minority and majority class, respectively.

By integrating the above overall weights with FCM-FSVM and KFCM-FSVM (simply replacing $\rho_{k}$ in equations (1) - (3) with $\omega_{k}^{+}$and $\omega_{k}^{-}$), the FCM-FSVM and KFCM-FSVM can reduce the effect of class imbalance in training dataset. The resultant FCM-FSVM and KFCM-FSVM integrated with the between-class weighting are termed as FCM-FSVM-W and KFCM-FSVM-W algorithm respectively in the remaining of the paper.

\subsection{RANDOM OVERSAMPLING}

Another approach adopted in this paper to handle the class imbalance problem is the random oversampling [15]. In this approach, the samples in the minority class are randomly redrawn with the replacement to create the new samples. Each new sample has the probability $\left(N_{m i}\right)^{-1}$ of being equally selected to any one of the original samples in the minority class, where $N_{m i}$ is the samples number of the minority class. The above generated samples will be added into the original samples to form a new minority class.

Adopting this approach for transformer fault diagnosis using a DGA dataset with the imbalanced class, the resampling is firstly performed on the minority class to increase the sample number of the minority class. And then FCMFSVM or KFCM-FSVM is invoked for fault classification. In the remaining of this paper, the FCM-FSVM and KFCMFSVM integrated with random oversampling are termed as FCM-FSVM-S and KFCM-FSVM-S algorithm, respectively. 


\section{CASE STUDIES}

This section provides case studies to evaluate the algorithms implemented in this paper, including FCM-FSVM and KFCM-FSVM, FCM-FSVM-W and KFCM-FSVM-W (i.e. FCM-FSVM and KFCM-FSVM integrated with between-class weighting), FCM-FSVM-S and KFCM-FSVM-S (i.e. FCMFSVM and KFCM-FSVM integrated with random oversampling). For the purpose of comparison, SVM, SVM-W (i.e. SVM integrated with between-class weighting), and SVM-S (i.e. SVM integrated with random oversampling) are also implemented for the case studies. In algorithmic implementations some software routines from the available toolbox have been adopted with necessary modifications and extensions [25].

\subsection{PARAMETERS SELECTION}

In the above algorithms, several common parameters are set as follows: the fuzziness parameter (equations 8 and 12) is set as $m=2$, the iterative termination value in FCM and KFCM is set as $\varepsilon=0.00001$ and the decay parameter (Equation 18) is set as $\beta=0.5$. For both FCM and KFCM, a random partition matrix is adopted for the initialization. Moreover, the radial basis function (RBF) kernel $K\left(\mathrm{x}_{i}, \mathrm{x}_{j}\right)=e^{-\gamma\left(\left\|\mathrm{x}_{i}-\mathrm{x}_{j}\right\|\right)^{2}}(\gamma$ is the variance parameter) is adopted for all the algorithms evaluated in the case studies.

In the case studies, the original DGA dataset is randomly split into two parts: a training dataset (consisting of about $70 \%$ samples of the original dataset) for deciding the hyperplane that can separate the samples into different classes (i.e. different fault types) and a testing dataset (consisting of about $30 \%$ of the original dataset) for verifying the classification accuracy of the algorithms. Note that the samples distribution among different classes in both training and testing dataset are kept as the same as that in the original dataset. To decide the optimal values of parameters $C$ and $\gamma$ in finding the separation hyperplane for the training dataset, a grid search with ten folds (or five folds) cross-validations are conducted on the training dataset for each of the above algorithms. As explained earlier, $C$ is the regularization parameter in FSVM to balance the margin maximization and classification violation (equation (1)) and $\gamma$ is the variance parameter of the RBF kernel.

Once the best set of parameters $C$ and $\gamma$ is found for each algorithm, each individual algorithm will be trained with its best set of parameters using the training dataset. Finally, the trained models (classifiers) are applied to assess the condition of transformers in the testing dataset. Each transformer in the testing dataset will be assigned a class label to indicate its condition, i.e. in normal operating condition or in any fault condition. Before running any algorithm, the data are normalized to $[0,1]$ interval. Two case studies are presented in the following.

\subsection{CASE STUDY}

The DGA dataset in the first case study is digested from Duval's IEC database [3] which consists of 70 DGA records. The faults occurred in the corresponding transformers include: discharge of low energy, discharge of high energy, low and medium temperature thermal fault, high temperature thermal fault, and partial discharge. As shown in Table 1, this dataset is randomly split into a training dataset (with 51 records) and a testing dataset (with 19 records).

In the dataset, most fault types have almost the same number of samples. Though the high energy discharge fault has more samples than other types of faults, the number difference is not much significant. Therefore, the above dataset will be treated as class balanced dataset and only SVM, FCM-FSVM and KFCM-FSVM algorithm are evaluated for transformer fault diagnosis based on this dataset. In these three algorithms, Duval's criteria are used as inputs, i.e. the input is the relative portion of three gases as follows: $\% \mathrm{C}_{2} \mathrm{H}_{2}=\mathrm{x} /(\mathrm{x}+\mathrm{y}+\mathrm{z}), \% \mathrm{C}_{2} \mathrm{H}_{4}=\mathrm{y} /(\mathrm{x}+\mathrm{y}+\mathrm{z})$, and $\% \mathrm{CH}_{4}=\mathrm{z} /(\mathrm{x}+\mathrm{y}+\mathrm{z})$, where $x, y$, and $z$ are gas concentrations of $\mathrm{C}_{2} \mathrm{H}_{2}, \mathrm{C}_{2} \mathrm{H}_{4}$, and $\mathrm{CH}_{4}$, respectively.

\begin{tabular}{l|c|c}
\multicolumn{3}{c}{ Table 1. DGA dataset I - total 70 records. } \\
\hline \multicolumn{1}{c|}{ Fault type } & $\begin{array}{c}\text { Training } \\
\text { Samples }\end{array}$ & $\begin{array}{c}\text { Testing } \\
\text { Samples }\end{array}$ \\
\hline Low energy discharge fault (D1) & 7 & 3 \\
\hline High energy discharge fault (D2) & 20 & 8 \\
\hline Lower/medium range thermal fault (T1) & 9 & 3 \\
\hline High range thermal fault (T2) & 9 & 3 \\
\hline Partial discharge (PD) & 6 & 2 \\
\hline Total & 51 & 19 \\
\hline
\end{tabular}

The procedure of dataset splitting, training and testing as described above was repeated ten times for SVM, FCMFSVM and KFCM-FSVM. The overall classification accuracy, which is defined as:

Overall Accuracy $=\frac{\text { number of correctly classified samples }}{\text { number of total samples }}$

is used as the performance indicator in this case study. Table 2 summarizes the accuracy on the testing dataset of the ten trials for each of the three algorithms.

In Table 2, the values of parameters $C$ (regularization parameter in FSVM) and $\gamma$ (variance parameter of radial basis function) are decided by conducting a grid search with ten folds cross-validation on the training dataset, where the pair $(\mathrm{C}, \gamma)$ is in the range of $\mathrm{C}=\left\{2^{0}, 2^{1}, \ldots 2^{10}\right\}$ and $\gamma=\left\{2^{-8}, 2^{-7}, \ldots\right.$ $2^{8}$. Moreover, since the Dataset I contain five faults types, the number of clusters in FCM and KFCM is set to five.

It can be seen from Table 2 that the averaged overall classification accuracy over ten trials of the three algorithms are KFCM-FSVM (92.6\%), FCM-FSVM (90.0\%), and SVM $(87.9 \%)$; and the best overall classification accuracy in ten trials of the three algorithms are KFCM-FSVM (100\%), FCMFSVM (94.7\%), and SVM (94.7\%). This indicates that KFCM-FSVM and FCM-FSVM outperform SVM, which is due to the introduction of weight for each sample in the training dataset in both KFCM-FSVM and KCM-FSVM to reduce the reverse effects of noises and outliers. Moreover, KFCM-FSVM is more robust and has tendency to attain 
higher classification accuracy than FCM-FSVM. This is because KFCM-FSVM algorithm maps the samples into a higher dimensional space before performing the clustering task.

Table 2. Classification accuracy of ten trials of algorithms (Dataset I).

\begin{tabular}{|c|c|c|c|c|}
\hline Algorithms & $\begin{array}{l}\text { Trial } \\
\text { No. }\end{array}$ & $\mathrm{C}$ & $\gamma$ & $\begin{array}{c}\text { Overall Classification } \\
\text { Accuracy }(\%) \\
\end{array}$ \\
\hline \multirow{11}{*}{ SVM } & 1 & 512 & 0.25 & 94.7 \\
\hline & 2 & 64 & 1 & 89.5 \\
\hline & 3 & 2 & 8 & 89.5 \\
\hline & 4 & 4 & 8 & 89.5 \\
\hline & 5 & 64 & 0.25 & 78.9 \\
\hline & 6 & 16 & 1 & 89.5 \\
\hline & 7 & 1024 & 0.0078125 & 84.2 \\
\hline & 8 & 4 & 2 & 84.2 \\
\hline & 9 & 256 & 1 & 84.2 \\
\hline & 10 & 1 & 8 & 94.7 \\
\hline & \multicolumn{3}{|c|}{ Average overall accuracy: } & $87.9( \pm 5.0)$ \\
\hline \multirow{11}{*}{ FCM-FSVM } & 1 & 512 & 0.125 & 94.7 \\
\hline & 2 & 16 & 0.5 & 89.5 \\
\hline & 3 & 64 & 1 & 89.5 \\
\hline & 4 & 8 & 8 & 89.5 \\
\hline & 5 & 128 & 8 & 84.2 \\
\hline & 6 & 16 & 4 & 89.5 \\
\hline & 7 & 4 & 1 & 89.5 \\
\hline & 8 & 64 & 8 & 89.5 \\
\hline & 9 & 512 & 0.03125 & 89.5 \\
\hline & 10 & 1024 & 4 & 94.7 \\
\hline & \multicolumn{3}{|c|}{ Average overall accuracy: } & $90.0( \pm 3.0)$ \\
\hline \multirow{11}{*}{ KFCM-FSVM } & 1 & 256 & 0.125 & 100 \\
\hline & 2 & 32 & 8 & 94.7 \\
\hline & 3 & 8 & 1 & 89.5 \\
\hline & 4 & 8 & 1 & 89.5 \\
\hline & 5 & 4 & 16 & 84.2 \\
\hline & 6 & 4 & 16 & 94.7 \\
\hline & 7 & 8 & 1 & 89.5 \\
\hline & 8 & 16 & 8 & 89.5 \\
\hline & 9 & 1 & 4 & 94.7 \\
\hline & 10 & 4 & 8 & 100 \\
\hline & \multicolumn{3}{|c|}{ Average overall accuracy: } & $92.6( \pm 5.1)$ \\
\hline
\end{tabular}

To test the significance of results of three algorithms as presented in Table 2, the t-test is conducted [26]. The t-test results show that the accuracy of three algorithms at significant level $\alpha=0.05$ are SVM $85.2 \%$, FCM-FSVM $88.4 \%$, and KFCM-FSVM $89.9 \%$.

Table 3 shows the typical classification results of both FCM-FSVM and KFCM-FSVM algorithm on 15 transformers. Due to the complexity of the ageing mechanism of insulation material, different fault types might co-exist in one transformer. Thus it would be useful to estimate the probabilities for each type of fault. In Table 3 the algorithm indicates the probability of each fault as a percentage. For example, KFCM-FSVM classification result shows transformer TX11 (Table 3) has about $63 \%$ probability of having T2 (high range thermal fault) and has about 25\% probability of having T1 (low to medium range thermal fault). Such interpretation will be beneficial for understanding the overall condition of a power transformer.
Table 3. KFCM-FSVM and FCM-KFCM Classification Results on 15 Transformers.

[D1 D2 T1 T2 PD] is the probability of each class, where D1- low energy discharge, D2- high energy discharge, T1- lower to medium range thermal fault, T2 - high range thermal fault, and PD - Partial discharge. [D1 D2 T1 T2 PD] ${ }^{1}$ is the classification result of KFCM-FSVM algorithm and [D1 D2 T1 T2 PD] $]^{2}$ is the classification result of FCM-FSVM algorithm.

\begin{tabular}{|c|c|c|c|}
\hline Transformer & $\begin{array}{c}\text { Probability } \\
\text { of Each Class (\%) }\end{array}$ & $\begin{array}{l}\text { Algorithms } \\
\text { Results }\end{array}$ & $\begin{array}{c}\text { On-site } \\
\text { Inspection }\end{array}$ \\
\hline TX1 & 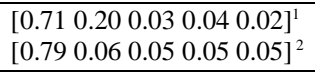 & $\begin{array}{l}\text { D1 } \\
\text { D1 }\end{array}$ & D1 \\
\hline TX2 & $\begin{array}{l}{\left[\begin{array}{lllll}0.72 & 0.17 & 0.04 & 0.04 & 0.03\end{array}\right]^{1}} \\
{\left[\begin{array}{lllll}0.53 & 0.38 & 0.03 & 0.03 & 0.03\end{array}\right]^{2}}\end{array}$ & $\begin{array}{l}\text { D1 } \\
\text { D1 }\end{array}$ & D1 \\
\hline TX3 & $\begin{array}{l}{\left[\begin{array}{llllll}0.93 & 0.01 & 0.02 & 0.02 & 0.02\end{array}\right]^{1}} \\
{\left[\begin{array}{lllll}0.50 & 0.41 & 0.03 & 0.03 & 0.03\end{array}\right]^{2}}\end{array}$ & $\begin{array}{l}\text { D1 } \\
\text { D1 }\end{array}$ & D1 \\
\hline TX4 & $\begin{array}{l}{\left[\begin{array}{llllll}0.06 & 0.88 & 0.02 & 0.03 & 0.01\end{array}\right]^{1}} \\
{\left[\begin{array}{lllll}0.09 & 0.80 & 0.05 & 0.03 & 0.03\end{array}\right]^{2}}\end{array}$ & $\begin{array}{l}\text { D2 } \\
\text { D2 }\end{array}$ & D2 \\
\hline TX5 & 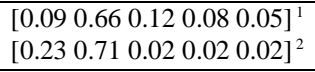 & $\begin{array}{l}\text { D2 } \\
\text { D2 }\end{array}$ & D2 \\
\hline TX6 & 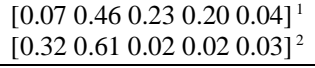 & $\begin{array}{l}\mathrm{D} 2 \\
\mathrm{D} 2\end{array}$ & D2 \\
\hline TX7 & 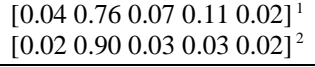 & $\begin{array}{l}\text { D2 } \\
\text { D2 }\end{array}$ & D2 \\
\hline TX8 & $\begin{array}{l}{\left[\begin{array}{lllll}0.06 & 0.90 & 0.01 & 0.02 & 0.01\end{array}\right]^{1}} \\
{\left[\begin{array}{lllll}0.03 & 0.83 & 0.04 & 0.06 & 0.04\end{array}\right]^{2}}\end{array}$ & $\begin{array}{l}\text { D2 } \\
\text { D2 }\end{array}$ & D2 \\
\hline TX9 & 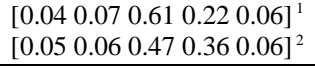 & $\begin{array}{l}\mathrm{T} 1 \\
\mathrm{~T} 1 \\
\end{array}$ & $\mathrm{~T} 1$ \\
\hline TX10 & $\begin{array}{l}{\left[\begin{array}{lllll}0.04 & 0.07 & 0.44 & 0.40 & 0.05\end{array}\right]^{1}} \\
{\left[\begin{array}{lllll}0.04 & 0.05 & 0.67 & 0.09 & 0.15\end{array}\right]^{2}}\end{array}$ & $\begin{array}{l}\mathrm{T} 1 \\
\mathrm{~T} 1\end{array}$ & $\mathrm{~T} 1$ \\
\hline TX11 & $\begin{array}{l}{\left[\begin{array}{lllll}0.04 & 0.06 & 0.25 & 0.63 & 0.02\end{array}\right]^{1}} \\
{\left[\begin{array}{lllll}0.05 & 0.06 & 0.21 & 0.62 & 0.06\end{array}\right]^{2}}\end{array}$ & $\begin{array}{l}\mathrm{T} 2 \\
\mathrm{~T} 2 \\
\end{array}$ & $\mathrm{~T} 2$ \\
\hline TX12 & 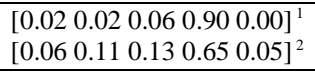 & $\begin{array}{l}\mathrm{T} 2 \\
\mathrm{~T} 2 \\
\end{array}$ & $\mathrm{~T} 2$ \\
\hline TX13 & $\begin{array}{l}{\left[\begin{array}{llllll}0.03 & 0.03 & 0.09 & 0.84 & 0.01\end{array}\right]^{1}} \\
{\left[\begin{array}{lllll}0.11 & 0.11 & 0.14 & 0.55 & 0.09\end{array}\right]^{2}}\end{array}$ & $\begin{array}{l}\mathrm{T} 2 \\
\mathrm{~T} 2\end{array}$ & $\mathrm{~T} 2$ \\
\hline TX14 & $\begin{array}{l}{\left[\begin{array}{lllll}0.05 & 0.05 & 0.26 & 0.06 & 0.58\end{array}\right]^{1}} \\
{\left[\begin{array}{lllll}0.05 & 0.06 & 0.26 & 0.08 & 0.55\end{array}\right]^{2}}\end{array}$ & $\begin{array}{l}\mathrm{PD} \\
\mathrm{PD}\end{array}$ & PD \\
\hline TX15 & $\begin{array}{l}{\left[\begin{array}{lllll}0.05 & 0.05 & 0.26 & 0.06 & 0.58\end{array}\right]^{1}} \\
{\left[\begin{array}{lllll}0.06 & 0.06 & 0.25 & 0.07 & 0.56\end{array}\right]^{2}}\end{array}$ & $\begin{array}{l}\mathrm{PD} \\
\mathrm{PD}\end{array}$ & PD \\
\hline
\end{tabular}

\subsection{CASE STUDY II}

This case study is to evaluate the performance of SVM, FCM-FSVM, KFCM-FSVM and their variants on a class imbalanced dataset. The DGA dataset used in the second case study consists of 368 DGA records, which are provided by a utility company. The corresponding transformers are either in normal operating condition or low to medium range thermal fault or partial discharge fault (Table 4).

Table 4. DGA dataset II - total 368 records.

\begin{tabular}{c|c|c}
\hline $\begin{array}{c}\text { Transformer } \\
\text { Condition }\end{array}$ & Training Samples & Testing Samples \\
\hline Normal & 141 & 60 \\
\hline Thermal Fault & 95 & 40 \\
\hline Partial Discharge & 23 & 9 \\
\hline Total & 259 & 109 \\
\hline
\end{tabular}

As shown in Table 4, this dataset exhibits unequal sample distribution among different fault types and can be regarded as a class imbalanced dataset: the samples in the class of normal condition significantly outnumber the samples in the class of partial discharge fault. For a class imbalance dataset, the overall classification accuracy (equation (21)), which is the portion of correctly classified samples, may not be a suitable 
performance indicator [24]. Instead, this paper adopts the geometric mean as the performance indicator for the class imbalanced dataset:

$$
\begin{gathered}
\mathrm{G}_{\mathrm{m}}=\sqrt{\mathrm{SE} \times \mathrm{SP}} \\
\mathrm{SE}=\frac{\text { number of correctly classified samples in majority class }}{\text { number of total samples in majority class }} \\
\mathrm{SP}=\frac{\text { number of correctly classified samples in minority class }}{\text { number of samples in minority class }}
\end{gathered}
$$

In this case study the procedure of dataset splitting, training and testing as mentioned earlier was repeated ten times on dataset II. The gas concentrations $\mathrm{C}_{2} \mathrm{H}_{2}, \mathrm{C}_{2} \mathrm{H}_{4}, \mathrm{C}_{2} \mathrm{H}_{6}, \mathrm{CH}_{4}$ and $\mathrm{H}_{2}$ in volume are used as inputs in the algorithms. Total nine algorithms including SVM, FCM-FSVM, KFCM-FSVM, SVM-W, FCM-FSVM-W, KFCM-FSVM-W, SVM-S, FCMFSVM-S, and KFCM-FSVM-S are evaluated using the above dataset in the case study.

In SVM-W, FCM-FSVM-W and KFCM-FSVM-W (i.e. SVM, FCM-FSVM, and KFCM-FSVM integrated with between-class weighting respectively), each sample in partial discharge fault class in training dataset is assigned with between-class weight of one, each sample in thermal fault class is assigned with between-class weight of $23 / 95=0.24$, and each sample in normal condition class is assigned with between-class weight of 23/141=0.16.

In SVM-S, FCM-FSVM-S and KFCM-FSVM-S (i.e. SVM, FCM-FSVM, and KFCM-FSVM integrated with random oversampling respectively), the number of samples in partial discharge class in both training and testing dataset is increased by three times using random oversampling. After random oversampling, the samples in each class are as follows: training dataset (normal class 141, thermal fault class 95, and partial discharge fault class 69); testing dataset (normal class 60 , thermal fault 40, and partial discharge class 27). The reason of only increasing the samples in partial discharge class by three fold is to minimize the noises and outliers, which might be introduced by the added samples. Table 5 summarizes the classification results (averaged over ten trials) of the above nine algorithms.

It can be seen from Table 5 that the overall classification accuracy could not accurately reflect the algorithm's performance on the class imbalanced dataset and may mislead the algorithm's validation. For example, for SVM-S, FCMFSVM-S, and KFCM-FSVM-S algorithm, the overall classification accuracy is $91.5 \%, 92 \%$, and $92.2 \%$ respectively. However, the SP (accuracy for the minority class) of these three algorithms is $73.8 \%, 80 \%$, and $82.6 \%$ respectively. In contrast, the geometric mean $\mathrm{Gm}$ of these three algorithms is $84.2 \%, 86.5 \%$, and $87.9 \%$ respectively, which could provide a reasonable performance indicator for the classification on both minority and majority class.

\begin{tabular}{|c|c|c|c|c|}
\hline Algorithm & $\begin{array}{c}\text { Overall } \\
\text { Classification } \\
\text { Accuracy }\end{array}$ & SE & SP & $\mathrm{Gm}$ \\
\hline SVM & 92.6 & 95.4 & 67.8 & 79.8 \\
\hline FCM-FSVM & 93.5 & 96.2 & 65.6 & 78.9 \\
\hline KFCM-FSVM & 94.4 & 96.3 & 73.4 & 83.8 \\
\hline SVM-W & 93.5 & 95 & 70 & 81.4 \\
\hline FCM-FSVM-W & 93.9 & 96.4 & 68.9 & 81.3 \\
\hline KFCM-FSVM-W & 94.6 & 96.7 & 74.5 & 84.5 \\
\hline SVM-S & 91.5 & 95.1 & 73.8 & 84.2 \\
\hline FCM-FSVM-S & 92 & 94.6 & 80 & 86.5 \\
\hline KFCM-FSVM-S & 92.2 & 93.8 & 82.6 & 87.9 \\
\hline
\end{tabular}

Table 5. Classification Results of Algorithms (Averaged over Ten Trials).

Table 5 shows that KFCM-FSVM, KFCM-FSVM-W, and KFCM-FSVM-S consistently outperform the corresponding SVM and FCM-FSVM, SVM-W and FCM-FSVM-W, and SVM-S and FCM-FSVM-S. This proves the consistence of the diagnosis capability of KFCM clustering based FSVM in the presence of measurement originated uncertainties. KFCMFSVM algorithm and its variants have the consistent tendency in achieving higher faults classification accuracy in the presence of noise and outliers as well as the class imbalance in the training dataset. Table 5 also indicates that the algorithms integrated with random oversampling approach can achieve better performance (in terms of geometric mean) than the algorithms integrated with between-class weighting approach in dealing with the effect of class imbalance in training dataset.

\section{CONCLUSIONS}

This paper presented a number of clustering based fuzzy support vector machine algorithms for DGA based transformer fault diagnosis under measurement originated uncertainties due to (a) the presence of noises and outliers and (b) the effect of class imbalance in a training dataset. The case studies presented in this paper indicate that compared to the standard support vector machine (SVM), the kernel fuzzy $c$-means fuzzy support vector machine (KFCM-FSVM) and its variants have consistent tendency to attain higher faults classification accuracy in power transformer fault diagnosis using DGA measurements.

\section{REFERENCES}

[1] IEEE Guide for the Interpretation of Gases Generated in Oil-Immersed Transformers, IEEE Standard C57.104-2008, 2009.

[2] M. Duval, "Dissolved gas analysis: it can save your transformer," IEEE Electr. Insul. Mag., Vol.5, No.6, pp. 22-27, 1989.

[3] M. Duval and A. dePablo, "Interpretation of Gas-In-Oil Analysis Using New IEX Publication 60599 and IEC TC 10 Databases", IEEE Electr. Insul. Mag., Vol. 17, No.2, pp. 31-41, 2001.

[4] C.F. Lin, J.M. Ling, and C.L. Huang, "An Expert System for Transformer Fault Diagnosis Using Dissolved Gas Analysis”, IEEE Trans. Power Delivery, Vol. 8, pp.1836-1841, 1993.

[5] M. Islam, T. Wu, and G. Ledwich, "A novel fuzzy logic approach to transformer fault diagnosis”, IEEE Trans. Dielectr. Electr. Insul., Vol. 7, pp. $177-186,2000$. 
[6] K. Tomsovic, M. Tapper, and T. Ingvarsson, "A Fuzzy Information Approach to Integrating Different Transformer Diagnostic Methods", IEEE Trans. Power Delivery, Vol. 11, pp. 1836-1841, 1996.

[7] J.L. Guardado, J.L. Naredo, P. Moreno, and C.R. Fuerte, "A Comparative Study of Neural Network Efficiency in Power Transformers Diagnosis Using Dissolved Gas Analysis", IEEE Trans. Power Delivery, Vol. 16, pp. 643- 647, 2001.

[8] Y. Zhang, X. Ding, Y. Liu, and P.J. Griffin, "An Artificial Neural Approach to Transformer Diagnostic Methods", IEEE Trans. Power Delivery, Vol. 8, pp. 1638-1644, 1993.

[9] V. Miranda and A.R.G. Castro, "Improving the IEC Table for Transformer Failure Diagnosis With Knowledge Extraction From Neural Network", IEEE Trans. Power Delivery, Vol. 20, pp. 2509-2516, 2005.

[10] Z.Y. Wang, Y.L. Liu and P.J. Griffin, "A Combined ANN and Expert System Tool for Transformer Fault Diagnosis", IEEE Trans. Power Delivery, Vol. 13, pp. 1224-1229, 1998.

[11] R. Naresh, V. Sharma, and M. Vashisth, "An Integrated Neural Fuzzy Approach for Fault Diagnosis of Transformers", IEEE Trans. Power Delivery, Vol. 23, pp. 2017-2024, 2008.

[12] Y.-C. Huang, H.-T. Huang, and C.-L. Huang, "Developing a New Transformer Fault Diagnosis System through Evolutionary Fuzzy logic", IEEE Trans. Power Delivery, Vol. 12, pp.761-767, 1997.

[13] Y.-C. Huang, "Evolving Neural Nets for Fault Diagnosis of Power Transformers", IEEE Trans. Power Delivery, Vol. 18, pp. 843-848, 2003.

[14] H. Ma, T. K. Saha, and C. Ekanayake, "Intelligent Framework and Techniques for Power Transformer Insulation Diagnosis", IEEE Power and Energy Society, General Meeting, Paper No. PESGM2009-000425, Calgary, Alberta, Canada, 2009.

[15] H. He and E.A. Garcia, "Learning from Imbalanced Data", IEEE Trans. Knowledge and Data Eng., Vol.21, pp. 1263-1284, 2002.

[16] V. Vapnik, The Nature of Statistical Learning Theory, Springer, 1995.

[17] N. Cristianini and J. Shawe-Taylor, An Introduction of Support Vector Machines and Other Kernel-Based Learning Methods, Cambridge University Press, 2001.

[18] C.-F. Lin and S.-D. Wang, "Fuzzy Support Vector Machines", IEEE Trans. Neural Networks, Vol.13, pp. 464-471, 2002.

[19] Y. Wang, S. Wang, and K.K. Lai, "A New Fuzzy Support Vector Machine to Evaluate Credit Risk," IEEE Trans. Fuzzy Systems, Vol.13, pp. 820-831, 2005.

[20] R. Babuska, P.J. van der Veen, and U. Kaymak, "Improved Covariance Estimation for GustafsonKessel Clustering”, IEEE Int'1. Conf. Fuzzy System, Honolulu, pp. 1081-1085, USA, 2002.

[21] M. Filippone, F. Camastra, F. Masulli, and S. Rovetta, "A Survey of Kernel and Spectral Methods for Clustering”, Pattern Recognition, Vol. 41, pp. 176-190, 2008.

[22] D. Graves and W. Pedrycz, "Kernel-based Fuzzy Clustering and Fuzzy Clustering: A Comparative Experimental Study", Fuzzy Sets and Systems, Vol. 161, pp. 522-543, 2010.

[23] X. Yang, G. Zhang, J. Lu, and J. Ma, "A Kernel Fuzzy c-Means Clustering-Based Fuzzy Support Vector Machine Algorithm for Classification Problems with Outliers or Noises", IEEE Trans. Fuzzy Syst., Vol. 19, pp. 105-115, 2011.

[24] R. Batuwita and V. Palade, "FSVM-CIL: Fuzzy Support Vector Machines for Class Imbalanced Learning”, IEEE Trans. Fuzzy Systems, Vol. 18, pp. 558-571, 2010.

[25] C.-C. Chang and C.-J. Lin, "LIBSVM: a library for support vector machines," available: http://www.csie.ntu.edu.tw/ cjlin/libsvm.

[26] D.V. Moore and G.P. Mccabe, Introduction to the Practice of Statistics, $5^{\text {th }}$ Edition, W.H. Freemand and Company, 2006.

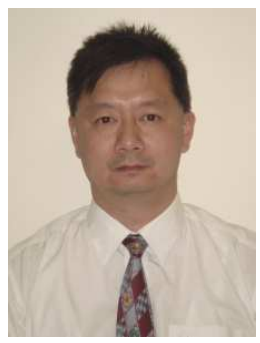

Hui Ma (M'95) received the B.Eng. and M.Eng. degrees from Xi'an Jiaotong University, China in 1991 and 1994, respectively, the M.Eng (by research) degree from Nanyang Technological University, Singapore in 1998, and the Ph.D. degree from the University of Adelaide, Adelaide, Australia in 2008. Currently Dr. Ma is a research fellow in the School of Information Technology and Electrical Engineering, the University of Queensland, Australia. Prior to joining the University of Queensland, Dr. Ma had many years of research and development experience. From 1994 to 1995, he was a researcher in Xi' an Jiaotong University, China. From 1997 to 1999, he worked as a firmware development engineer in CET Technologies Pte. Ltd., Singapore. He was with Singapore Institute of Manufacturing Technology as a research engineer from 1999 to 2003. His research interests include industrial informatics, condition monitoring and diagnosis, power systems, wireless sensor networks, and sensor signal processing.

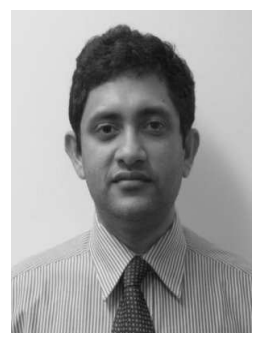

Chandima Ekanayake (M'00) received the B.Sc.Eng.(Hons) degree in 1999 from the University of Peradeniya, Sri Lanka. He obtained the Tech. Lic. and $\mathrm{Ph} . \mathrm{D}$. degrees from Chalmers University of Technology Sweden in 2003 and 2006, respectively. Currently he is a lecturer in the School of Information Technology and Electrical Engineering, the University of Queensland (UQ), Brisbane, Australia. Before joining UQ he was with the University of Peradeniya Sri Lanka as a Senior lecturer. During his Ph.D. degree studies he was working for a European Union Project called REDIATOOL where he engaged in research related to diagnostics of transformer insulation from dielectric response measurements. From 2001, he has been involving on condition monitoring of transformers installed at Ceylon Electricity Board, Sri Lanka. He was the Chair of IEEE Sri Lanka Section in year 2006 and 2007. His research interests are condition monitoring of power apparatus, alternatives for insulating oil, transient studies on power systems and energy related studies.

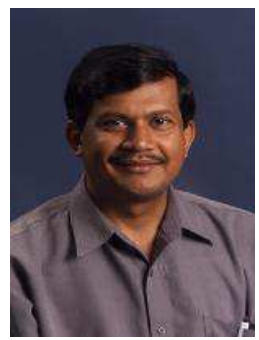

Tapan Kumar Saha (M'93-SM'97) was born in Bangladesh in 1959 and immigrated to Australia in 1989. He received the B.Sc. Eng. (electrical and electronic) in 1982 from the Bangladesh University of Engineering \& Technology, Dhaka, Bangladesh, M. Tech (electrical engineering) in1985 from the Indian Institute of Technology, New Delhi, India and the Ph.D. degree in 1994 from the University of Queensland, Brisbane, Australia. Tapan is currently Professor of Electrical Engineering in the School of Information Technology and Electrical Engineering, University of Queensland, Australia. Previously he has had visiting appointments for a semester at both the Royal Institute of Technology (KTH), Stockholm, Sweden and at the University of Newcastle (Australia). He is a Fellow of the Institution of Engineers, Australia. His research interests include condition monitoring of electrical plants, power systems and power quality. 